\title{
IMPACTO PSICOSSOCIAL EM TRANSPLANTE HEPÁTICO
}

\author{
Psychosocial impact on liver transplantation
}

\author{
Clerison Garcia',2, Ehideé La Rotta ${ }^{3}$, Ilka Boin ${ }^{4}$, Cássia Garcia ${ }^{5}$, Agnaldo Lima ${ }^{6}$
}

\section{RESUMO}

Nas últimas décadas, estudos sobre o impacto psicossocial e transplante de órgãos e tecidos têm sido realizados; porém pode-se perceber que são escassas as pesquisas que avaliam os aspectos psicossociais ou o impacto psicossocial no processo do transplante hepático, principalmente entre adultos. O objetivo deste estudo foi apresentar e discutir os achados na literatura referentes aos impactos psicossociais presentes no transplante de fígado. Realizou-se revisão da literatura que compreendeu o período entre 2010 e outubro de 2016, nas bases de dados Medline e Lilacs/Bireme, e nas bibliotecas eletrônicas Scielo e Google Acadêmico, utilizando-se como descritores: impacto psicossocial, transplante de fígado e psicologia. Foram selecionadas onze publicações por cumprirem os critérios de inclusão, tendo sido identificados que oito pesquisavam o impacto ou aspectos psicossociais em crianças e adolescentes candidatos a transplante e transplantados e/ou as reações parentais, e somente três artigos em pacientes adultos. Conclui-se que os estudos que avaliam impacto psicossocial em pacientes adultos (que evoluem com doenças crônicas hepáticas em estágio avançado e que requerem transplante hepático), assim como em seus familiares são poucos, considerando-se a grande necessidade de melhorar a avaliação e o atendimento psicológico dessa clientela.

Descritores: Impacto Psicossocial; Transplante de Fígado; Psicologia.

Instituições:

${ }^{1}$ Programa de Pós-Graduação em Ciências aplicadas à Cirurgia Faculdade de Medicina - Universidade Federal de Minas Gerais, Belo Horizonte/MG, Brasil.

${ }^{2}$ Programa de Pós-Graduação em Ciências aplicadas a Cirurgia Universidade Estadual de Campinas, Campinas/SP, Brasil.

${ }^{3}$ Programa de Pós-Graduação em Saúde Coletiva - Universidade Estadual de Campinas, Campinas/SP, Brasil.

${ }^{4}$ Unidade de Transplante Hepático - Hospital das Clínicas Universidade Estadual de Campinas, Campinas/SP, Brasil.

${ }^{5}$ Programa de Pós-Graduação em Gerontologia- Universidade Estadual de Campinas, Campinas/SP, Brasil.

${ }^{6}$ Grupo de Transplante do Instituto Alfa de Gastroenterologia Hospital das Clínicas - Universidade Federal de Minas Gerais, Belo Horizonte/MG, Brasil.

Correspondência:

Clerison Stelvio Garcia

Av.Maria Emília A S de Angelis, 730/82, CEP 13044-163 - Campinas /SP

Tel.: (19) 98358-2830

E-mail: clerison_garcia@yahoo.com.br

Recebido em: 13/12/2016

Aceito em: 13/01/2017

\section{INTRODUÇÃO}

O diagnóstico da doença crônica, especialmente aquela que pode levar à morte, é extremamente doloroso e traz mudanças drásticas no modo de vida, na rotina, nos pensamentos e nas emoções de pacientes de diferentes idades e do grupo familiar. ${ }^{1,2}$

Apesar de muitas vezes o transplante ser a única terapêutica que pode aumentar a sobrevida e melhorar a qualidade de vida de pacientes com doenças crônicas, ${ }^{3}$ é caracterizado como importante estressor na vida desses indivíduos e de seus familiares. Dessa forma, é importante observar os aspectos psicossociais que revelam os sentimentos e condutas semelhantes ou desafiadoras em doenças crônicas graves com risco de morte. ${ }^{4}$

Estudos apontam que crianças transplantadas podem apresentar distúrbios emocionais, comportamentais e sociais, com maior significância. 5 Outros estudos descrevem os problemas psicossociais presentes no 
transplante hepático, como sintomas psiquiátricos e emocionais. ${ }^{2,5}$ Os problemas psicológicos e psiquiátricos são diversos, desde transtornos de ajustamento transitório até depressão maior e transtorno de estresse pós-traumático, sendo depressão e ansiedade os mais prevalentes. ${ }^{4,5}$

Os sintomas psicológicos encontrados com maior frequência em transplantados em geral são: problemas de identidade, baixa autoestima, isolamento, inibição das emoções, depressão e altos níveis de ansiedade. , $^{2,5,6}$

Os problemas psicossociais descritos entre crianças e adultos com indicação de transplante, em especial de fígado, são: ajustamento familiar, social e individual, dificuldades de relacionamento, problemas de adesão ao tratamento, insegurança, problemas com pensamentos e atenção, comportamento delinquente e agressividade..$^{5,7-9}$

O impacto do transplante no paciente e na família pode afetar e influenciar o funcionamento do sistema familiar e ainda modificar a atuação individual dos membros familiares ou, até interferir no sistema como um todo, exatamente pelas necessidades complexas de cuidados exigidas nessa modalidade terapêutica. . $^{8,10,11}$

A experiência do transplante pode produzir sentimento de caos 2 e desorganizar profundamente a vida do paciente e da sua família, forçando-lhes a fazer ajustamentos. 5 A rotina torna-se extenuante, com consultas frequentes ao médico, ingestão de medicamentos, exames e internações hospitalares. ${ }^{8,12}$

A ausência de comunicação entre os familiares sobre o transplante (seja por razões culturais ou psicológicas) pode dificultar a elaboração psíquica da experiência de transplante, podendo tornar-se um processo silencioso e solitário. . $^{13-15}$

A omissão de informações foi relatada por Gritti et al, 2006, como causa de problemas psicológicos em longo prazo em crianças e adolescentes transplantados, com idade entre cinco e 14 anos. Dados da pesquisa sugeriram que, quando o transplante é um segredo na família, pode tornar-se uma experiência obscura e traumática para a criança. ${ }^{13}$

Nas últimas décadas, têm-se realizado estudos sobre o impacto psicossocial e transplante, porém são escassas as pesquisas que avaliam os aspectos psicossociais ou o impacto psicossocial no processo de transplante hepático, desde o diagnóstico da doença irreversível, indicação de transplante, período de espera até o póstransplante imediato e tardio. ${ }^{3,4}$

Este tema é relevante para a prática profissional, considerando que cada etapa do processo de transplante tem sua singularidade e necessidade específicas, nas dimensões psicológica, social e multidisciplinar. ${ }^{4}$
Dessa forma, o objetivo deste estudo foi apresentar e discutir os achados da literatura referentes aos impactos psicossociais presentes no transplante de fígado.

\section{MÉTODO}

O presente estudo trata-se de uma revisão narrativa da literatura, ${ }^{16}$ para a qual buscaram-se artigos publicados no período entre 2010 e 2016, realizado nas bases de dados e fontes eletrônicas Medline, Lilacs/Bireme, Scielo, e Google Acadêmico, utilizando-se como descritores: impacto psicossocial, transplante de fígado e psicologia.

Foram encontrados 265 artigos. Após a leitura dos títulos e dos resumos, selecionaram-se onze publicações para a leitura na íntegra, que cumpriram com os critérios de inclusão: artigos originais ou dissertações/teses que se relacionavam com os três descritores definidos, em português ou inglês, realizados com indivíduos de todas as faixas etárias, durante o período pré e póstransplante hepático, publicados entre 2010 e 2016. Foram excluídos artigos que não se relacionavam com os três descritores e as pesquisas que foram publicadas fora do período de 2010 a 2016.

\section{RESULTADO e DISCUSSÃO}

Dos artigos selecionados, oito estudavam a população infantil e adolescente, avaliando os impactos psicossociais do transplante de fígado nesses indivíduos. ${ }^{13,17-23}$ Observouse também, que cinco das publicações selecionadas eram teses ou dissertações, sendo que isso se justifica, pois discutir impacto psicossocial em transplante hepático é tema recente. Dentro dos departamentos de transplante, normalmente debatem-se com frequência as técnicas cirúrgicas, medicamentos de imunossupressão e condutas clínicas do transplante hepático. No entanto, é importante avaliar, também, aspectos psicossociais, emocionais e relação interpessoal dos candidatos ao transplante, pois favorecem o pré e o pós-transplante de fígado e a sobrevida dos pacientes, além de minimizar o impacto na família. ${ }^{4}$

Apenas três estudos referiam-se à população adulta, o que demonstra a necessidade de pesquisas com esses indivíduos em relação à temática apresentada, visto a importância desta na terapêutica do transplante. ${ }^{4,24,25}$

No transplante hepático existem dois princípios básicos. O primeiro é de natureza social: sem doação não há transplante. O segundo é de natureza médica: o transplante não inicia nem termina no processo cirúrgico em si. Há quatro fases no transplante: a 
fase pré-transplante, o transplante, o retorno à vida funcional e a evolução em longo prazo, cada uma com suas particularidades, e em todas essas etapas há a necessidade de um acompanhamento multiprofissional. ${ }^{26}$ Todo o processo de transplante hepático é dinâmico e relacional e necessita de um sistema agregador entre paciente-família-equipe; é um processo complexo que precisa da adesão do paciente e da família. ${ }^{16,22,27,28}$

\section{Impacto psicossocial em crianças, adolescentes e familiares}

Os efeitos do transplante hepático na função psicológica e cognitiva são, ainda, pouco claros, necessitando-se de pesquisas longitudinais. ${ }^{29}$ Contudo, constatam-se as consequências psicológicas do processo de transplante em crianças e adolescentes. Quanto a aspectos sociais, foram verificados: isolamento social, baixo nível educacional e pouca independência. Esses efeitos podem ter colaborado para a não aderência do paciente ao tratamento pós-transplante (incluindo a recusa a tomar medicamentos), resultando em retransplantes e menores taxas de sobrevida entre os que realizaram o transplante na idade entre 12 e 17 anos, considerando que a puberdade é o período de desenvolvimento físico e psicológico ao qual compete o crescimento físico e a maturidade intelectual. ${ }^{22}$ Neste sentido, a atenção da equipe multidisciplinar ao identificar as barreiras para a aderência ao tratamento é crucial para melhorar o tratamento do paciente adolescente, ${ }^{22}$ considerando que outro estudo verificou uma tendência para jovens e adolescentes apresentarem melhor qualidade de vida quanto mais cedo realizarem o transplante, apesar de isso parecer melhorar à medida em que for decorrendo o tempo após o transplante (11 anos ou mais). ${ }^{20}$

Outro estudo com adolescentes entre 12 e 18 anos, que realizaram transplante de fígado, verificou que a qualidade de vida desses indivíduos apresentouse pior em quase todos os domínios, comparando-se com a população adolescente em geral. Alguns efeitos colaterais que ocorrem após o transplante, como dores de cabeça, ganho de peso, dores nas articulações e dificuldades para dormir poderiam interferir na presença de sintomas angustiantes e, consequentemente, na pior qualidade de vida desses adolescentes. ${ }^{23}$ Observouse também forte associação negativa entre tratamento psicológico pregresso e o domínio psicológico de qualidade de vida, sendo que o acompanhamento psicológico foi realizado somente um período após o transplante hepático entre a maioria dos pacientes, e havia poucas informações sobre sua história de saúde mental antes do transplante. ${ }^{23}$

Segundo Gritti et al, 2013, as crianças submetidas ao processo de transplante hepático têm maior propensão a sofrer problemas psicossociais e psicológicos no período pré e pós-transplante. ${ }^{13}$ A necessidade de transplante, como única alternativa terapêutica e a espera por um doador, que por vezes é longa, acarretam sofrimento psicológico ao paciente e aos seus familiares, que devem adaptar-se a condições altamente estressoras .22

Da mesma forma, outra investigação com mães de crianças transplantadas, na idade entre quatro e oito anos, verificou os aspectos psicossociais das diferentes fases do processo de transplante em famílias vivendo diferentes períodos pós-transplante, tendo relatado a importância da qualidade da comunicação por parte da equipe de saúde, com empatia e sensibilidade, considerando o forte impacto da notícia da necessidade de transplante, sendo algo assustador para os familiares

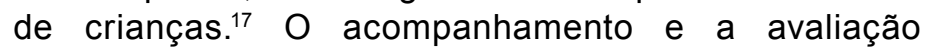
psicológicos da criança e da família no período prétransplante são importantes para detectar e trabalhar possíveis problemas psicossociais, prevenindo dessa forma, intercorrências futuras, considerando que choque, desespero, perda de referenciais e impotência foram sentimentos comuns entre as mães. A presença de um profissional para fornecer informações sobre o paciente durante a cirurgia foi questão que se mostrou deficiente e, no período pós-transplante, também se mostrou a importância do acompanhamento dessas famílias, tendo em vista que algumas mães relataram demorar mais de três anos para se tranquilizarem e se habituarem com a rotina de cuidados. ${ }^{17}$ Já no período pré-transplante, uma pesquisa verificou que $65,7 \%(n=25)$ dos pais avaliados foram diagnosticados com doença psiquiátrica, sendo que destes, sete apresentavam depressão e dezoito, desordens de ansiedade (sintomas que foram mais prevalentes entre as mães), concluindo que se torna necessária a avaliação psicossocial dos pais das crianças com doenças crônicas e, no caso, candidatas a transplante, como parte da rotina de cuidados, para que sejam realizadas intervenções precoces, quando necessário. Nesse sentido, a adequada saúde mental da família, desde os estágios iniciais da doença, pode interferir positivamente na saúde da criança. ${ }^{30,31}$

Ainda em relação aos aspectos psicossociais dos pais, quando eles se tornam doadores de seus filhos pode haver uma tentativa de reduzir alguns dos efeitos perturbadores da doença, oferecendo uma chance de sobrevida com maior qualidade à criança. O processo de tratamento da doença pode impactar a família, com diminuição das relações sociais, aumento de problemas entre cônjuges e de relacionamentos entre os irmãos das crianças doentes, devido à sobrecarga emocional e física do momento em que vivem, o que deve ser levado em consideração, incentivando o diálogo entre o casal, com o objetivo de manter o sistema familiar equilibrado, 
visto que o resultado da cirurgia e o prognóstico são otimizados quando existe unidade familiar. ${ }^{18,31} \mathrm{Em}$ pesquisa realizada com pais e crianças portuguesas, verificaram-se índices de sintomatologia depressiva mínimos entre os pais, além de que as famílias que frequentaram mais vezes ao ano o hospital apresentavam maior impacto na dinâmica familiar, tendo por sua vez, aumentado o stress e sintomatologia depressiva. O estudo verificou também que, quando os pais apresentavam níveis de stress mais altos, os filhos tendiam a apresentar níveis de autoconceito mais baixos. ${ }^{19}$

Outro estudo português, que avaliou crianças e adolescentes de cinco a 18 anos, no pré e póstransplante hepático, observou que esses indivíduos apresentaram fragilidades emocionais, afetivas e intelectuais, em qualquer fase da doença. Observouse um funcionamento mental pautado, sobretudo, por imposição da racionalidade, em prol da repressão dos afetos e emoções (alexitemia), o que poderia repercutir em pobreza criativa e prejuízo ao nível das performances cognitivas, sendo que, um processo de estímulo da expressão verbal do que o paciente sente, poderia repercutir não somente na melhora do rendimento escolar, mas também na saúde geral dessas crianças e adolescentes. O mesmo estudo ainda cita a importância da avaliação psicossocial pré-transplante, como propósito de se conhecer os potenciais problemas e riscos capazes de prejudicar a recuperação e que podem surgir durante a terapêutica. ${ }^{21}$

Corroborando estes dados, Suris et al, 2004, afirmou que adolescentes que têm doenças crônicas podem apresentar atraso na maturidade e infantilização, demora na aquisição de autonomia e déficit no desenvolvimento cognitivo. ${ }^{32}$

Ao avaliar o impacto do transplante hepático em crianças e adolescentes entre quatro e 16 anos, Freitas, 2013, observou que "a situação profissional da mãe" e "nível educacional da mãe" foram relacionados, de forma significativa, à melhora da qualidade de vida e autoconceito da amostra estudada, assim como uma maior autoestima e maior autoconceito a nível intelectual quando as mães se encontravam empregadas, mostrando que as crianças e adolescentes sentiamse seguros quanto aos seu rendimento obtido nas tarefas intelectuais e capacidade de aprendizagem, o que mostra que o nível educacional das mães poderia interferir no estado intelectual, bem-estar e auto-estima de seus filhos no pós-transplante hepático. ${ }^{19}$

\section{Impacto psicossocial em adultos}

Felício, 2007, enumera algumas preocupações e situações estressantes da realidade de um candidato ao transplante hepático, como: o medo relativo à integridade física e psicológica, as incertezas quanto à evolução da doença e resultado da cirurgia, as complicações que podem ocorrer no período pós-cirúrgico, a apreensão e a insegurança relacionadas ao fígado que deverá ser substituído, os extensos encargos financeiros e a preocupação quanto ao futuro pessoal, familiar e social. Além disso, os contatos com o sistema de saúde tendem a desencadear uma trilogia de reações psicológicas: baixa autoestima, ansiedade e depressão. ${ }^{27}$

Dentre pacientes adultos com cirrose hepática de qualquer etiologia, verificou-se que, no período prétransplante, os sentimentos de mal-estar, tristeza e a limitação da independência têm forte representação na vida desses indivíduos, assim como a incapacidade para trabalhar e alteração das relações sociais. ${ }^{24}$ No entanto, são os sentimentos de expectativa, esperança e espiritualidade que auxiliam o doente a enfrentar a doença no período pré-transplante, e são determinantes na forma como o indivíduo vive e sente sua capacidade. Pelo fato desses pacientes muitas vezes apresentarem suporte social somente na família, é nos profissionais de saúde que eles encontram também o suporte de que necessitam, considerando satisfatórias: a relação de ajuda, humanização dos cuidados, apoio e atenção dispensados, o que poderia também diminuir o sofrimento, aumentar a adesão aos comportamentos saudáveis e tratamentos sugeridos, bem como diminuir a possibilidade de abandono dos cuidados. ${ }^{24}$ Apesar disso, os pacientes citaram que nenhum apoio psicológico ou um compartilhar de experiências com doentes transplantados Ihes foi proposto no período pré-transplante, o que seria importante, já que muitos apresentam ansiedade/depressão, nessa fase do tratamento. No pós-transplante, verificou-se que os sentimentos de ansiedade e depressão foram substituídos por alegria e satisfação, assim como, após uma ano de transplante, que houve melhoria acentuada na autoestima. ${ }^{24}$

O transplante acontece no individuo que é constituído de história, memórias, sentimentos, pensamentos e identidade. Porém, traz a controvérsia de possuir o próprio órgão depauperado e a recuperação da vida através do órgão saudável e quase sem vida. Todo esse entendimento leva um tempo, não cronológico, para fazer sentido e vai depender do impacto emocional vivido no processo de transplante do fígado. ${ }^{33}$

O paciente é arrancado de seu cotidiano e é lançado à rotina extenuante de consultas médicas e avaliações, exames de rotina complexos e invasivos, regularização dos medicamentos e, muitas vezes, internações. ${ }^{6,34}$ 
Essa mudança repentina e intensa tem sido identificada como a variável que compromete, interfere e afeta o funcionamento e o bem-estar do sistema familiar. ${ }^{35,36}$

Sendo assim, faz-se necessária a alteração comportamental, cognitiva, emocional e social, para a boa adaptação e, também, para afetar o menos possível a qualidade de vida. ${ }^{26}$

A avaliação e o acompanhamento psicológico em transplante hepático exigem tanto do cirurgião transplantador quanto do profissional psicólogo e da equipe multidisciplinar. ${ }^{37} \mathrm{~A}$ linha que os une é a imensa complexidade de intervir, a exigência de ponderação e o rigor técnico preciso. O ponto divergente é que a intervenção psicológica é menos invasiva, menos radical e mais conservadora. ${ }^{38,39}$

No período pré-transplante, a avaliação e o acompanhamento psicológico deveriam ser procedimentos obrigatórios, preenchendo a lacuna que clareia os possíveis riscos emocionais, sociais, afetivos e psicológicos, além de fortalecer o candidato para o procedimento cirúrgico..$^{4,40}$

A avaliação e o acompanhamento psicológico em candidatos pré-transplante têm por objetivo identificar o histórico de problemas psicológicos e suas relações interpessoais, moderar as expectativas, desenvolver a capacidade de adaptação a situações estressantes, avaliar o nível de suporte social e o impacto na família, avaliar a necessidade do processo de terapia psicológica e, principalmente, aceitar o transplante como procedimento fundamental para manter a vida.4,38,40

Após a concretização do tão esperado transplante, há necessidade de enorme atenção, pela complexidade e recuperação da saúde. Nessa volta à vida, o pósoperatório imediato é período de alterações, com desconforto físico e sensações emocionais profundas para o paciente e toda a família. ${ }^{40}$

Nesse período, o estado emocional do paciente passa por instabilidade e alguns sintomas podem surgir, sendo que os mais frequentes são: ansiedade, depressão e alucinações, o que pode estar relacionado com a terapia imunossupressora e o ajuste da dosagem de medicamentos como os corticosteroides. ${ }^{41}$

Outras dificuldades presentes na fase do pósoperatório são: dificuldades de ajustamento, sintomas psicopatológicos, não adesão ao plano terapêutico e instabilidade emocional. .,41,42 $^{9}$

No estudo de Corruble, 2011, há relato de aumento dos sintomas depressivos no processo de transplante hepático, desde inserção e permanência na lista de espera até o pós-transplante, que estão associados a um risco aumentado de mortalidade, em longo prazo. ${ }^{25}$
Assim, a avaliação e o acompanhamento psicológico durante as fases de pré e pós-transplante devem ser valorizados pelas equipes transplantadoras, devido às importantes consequências na vida e saúde do paciente e de sua família. Anton, 2010 e Gruttadauria, 2009, relataram a importância de identificar e reduzir fatores de risco nos aspectos psicossociais, psicológicos e psiquiátricos, para o sucesso integral do transplante hepático. ${ }^{17,42}$

Sendo assim, torna-se indispensável na equipe de transplante, um profissional psicólogo, com especialização ou aprimoramento na área, para encorajar o doente a ser transplantado, auxiliar a equipe transplantadora quanto à interpretação dos comportamentos e medos dos pacientes, selecionar as informações adequadas para a equipe, analisar as relações presentes entre os candidatos e seus familiares, além de criar e fortalecer os recursos mentais do próprio doente e auxiliá-los a entender que muitos sintomas, como as alucinações, são provocados pelos imunossupressores. ${ }^{38,41}$

\section{CONCLUSÃO}

Observa-se a necessidade de novos estudos que avaliem e discutam o tema impacto psicossocial em transplante hepático, principalmente entre adultos.

As crianças e adolescentes que apresentam maior impacto psicossocial demonstraram maior isolamento social, atraso na maturidade, menor independência e pior qualidade de vida.

Em adolescentes verificou-se preocupação em relação ao crescimento físico e maturidade intelectual.

O maior impacto psicossocial com mães de crianças pré e pós-transplante foi a presença de depressão e ansiedade.

Adultos candidatos a transplante hepático com maior impacto psicossocial apresentaram altos índices de depressão, ansiedade e baixa autoestima. Na fase póstransplante o maior impacto psicossocial relacionouse à instabilidade emocional, não adesão ao plano terapêutico e sintomas psicopatológicos.

A avaliação e o acompanhamento psicológico durante as fases de pré e pós-transplante pode diminuir o impacto psicossocial.

O profissional psicólogo deve ser valorizado pelas equipes transplantadoras para o sucesso integral do transplante hepático. 


\section{ABSTRACT}

In the last decades, studies have been made on the psychosocial impact and transplantation; however, it can be noticed that the researches assessing the psychosocial aspects or the psychosocial impact in the process of liver transplantation are scarce, especially among adults. Therefore, the purpose of this study was to present and discuss the findings in the literature regarding the psychosocial impacts of the liver transplantation. A review of the literature published between 2010 and 2016 in the databases Medline and Lilacs / Bireme and electronic sources Scielo and Google Scholar was conducted using as keywords: psychosocial impact, liver transplantation and psychology. Eleven articles were selected to meet the inclusion criteria, identifying eight articles that investigated the impact or psychosocial aspects in liver transplantation candidates or transplanted children and adolescents or their parents. Only three articles focused adult patients It can be concluded that studies assessing the psychosocial impact in adult patients affected with severe chronic hepatic diseases requiring liver transplantation and their relatives are few, and it is necessary to carry out studies with such aim, with the purpose to improve the psychological care of that group. In the last decades, studies have been made on the psychosocial impact and transplantation; however, it can be noticed that the researches assessing the psychosocial aspects or the psychosocial impact in the process of liver transplantation are scarce, especially among adults. Therefore, the purpose of this study was to present and discuss the findings in the literature regarding the psychosocial impacts of the liver transplantation. A review of the literature published between 2010 and 2016 in the databases Medline and Lilacs / Bireme and electronic sources Scielo and Google Scholar was conducted using as keywords: psychosocial impact, liver transplantation and psychology. Eleven articles were selected to meet the inclusion criteria, identifying eight articles that investigated the impact or psychosocial aspects in liver transplantation candidates or transplanted children and adolescents or their parents. Only three articles focused adult patients It can be concluded that studies assessing the psychosocial impact in adult patients affected with severe chronic hepatic diseases requiring liver transplantation and their relatives are few, and it is necessary to carry out studies with such aim, with the purpose to improve the psychological care of that group.

Keywords: Psychosocial Impact; Liver Transplantation; Psychology.

\section{REFERÊNCIAS}

1. de Oliveira ÉA, dos Santos MA, Mastropietro AP, Picini VS, Bueno CC, Matos RN, et al. Grupo de apoio ao familiar do transplantado de medula óssea. Revista da SPAGESP. 2003;4(4).

2. Wise BV. In their own words: The lived experience of pediatric liver transplantation. Qualitative Health Research. 2002;12(1):7490.

3. Mies S. Transplante de Fígado. Rev Ass Med Brasil 1998;44(2):127-34.

4. Garcia CS. O suporte social e o impacto na família de pacientes candidatos a transplante de fígado. Belo Horizonte, Brasil: Universidade Federal de Minas Gerais; 2013.

5. Engle D. Psychosocial aspects of the organ transplant experience: what has been established and what we need for the future. Journal of clinical psychology. 2001;57(4):521-49.

6. Fine RN, Alonso EM, Fischel JE, Bucuvalas JC, Enos RA, Gore-Langton RE. Pediatric transplantation of the kidney, liver and heart: summary report. Pediatric transplantation. 2004;8(1):75-86.

7. Griffin KJ, Elkin TD. Non-adherence in pediatric transplantation: A review of the existing literature. Pediatric transplantation. 2001;5(4):246-9.
8. Falkenstein K. Proactive psychosocial management of children and their families with chronic liver disease awaiting transplant. Pediatric transplantation. 2004;8(3):205-7.

9. Shemesh E. Assessment and management of psychosocial challenges in pediatric liver transplantation. Liver Transplantation. 2008;14(9):1229-36.

10. Vega-Angarita O, González-Escobar D. Apoyo social: elemento clave en el afrontamiento de la enfermedad crónica. Enfermería global. 2009;8(2).

11. Oliveira ÉA, Santos MA, Mastropietro AP, Picini VS, Bueno CC, Matos RN, et al. Grupo de apoio ao familiar do transplantado de medula óssea. Revista da SPAGESP. 2003;4(4):52-62.

12. Castro EK, Piccinini CA. A experiência de maternidade de mães de crianças com e sem doença crônica no segundo ano de vida. Estudos de psicologia. 2004;9(1):89-99.

13. Gritti A, Sicca F, Di Sarno AM, Di Cosmo N, Vajro S, Vajro P. Emotional and behavioral problems after pediatric liver transplantation: A quantitative assessment. Pediatric transplantation. 2006;10(2):205-9.

14. Chaib E, Figueira ERR, Brunheroto A, Gatti AP, Fernandes DV, D'Albuquerque LAC. A seleção de pacientes utilizandose o critério meld melhora a sobrevida a curto prazo dos pacientes submetidos ao transplante de fígado? ABCD Arquivos Brasileiros de Cirurgia Digestiva (São Paulo). 2013;26(4):324-7. 
15. Aguiar MIF, Ribeiro Mariano M, Verlaine Oliveira R, Augusta Batista V. Existência Humana e Desafios Éticos no Cuidado ao paciente à Espera de Transplante Hepático. Journal of Nursing UFPE/Revista de Enfermagem UFPE. 2013;7(11).

16. Rother, ET. Revisão sistemática $X$ revisão narrativa. Acta Paul. Enferm. 2007;20(2):v-vi.

17. Anton MC, Piccinini CA. Aspectos psicossociais associados a diferentes fases do transplante hepático pediátrico. Psicologia: teoria e pesquisa. 2010;26(3):455-64.

18. Vasconcelos APL. Pais doadores no transplante hepático pediátrico. São Paulo: Universidade de São Paulo; 2014.

19. Freitas SFM. Impacto do transplante hepático pediátrico na dinâmica familiar. Coimbra: Universidade de Coimbra, Faculdade de Psicologia e de Ciências da Educação; 2013.

20. Gouveia MJ. Impacto do transplante hepático pediátrico na qualidade de vida pós-transplante: estudo exploratório. Coimbra: Universidade de Coimbra; 2013.

21. Assunção CP. Dinâmica afectiva e performance intelectual em crianças e adolescentes submetidos a transplante hepático e com insuficiência hepática crónica: estudo exploratório. Coimbra: Universidade de Coimbra; 2015.

22. Samyn M. Optimizing outcomes for pediatric recipients. Liver Transplantation. 2012;18(S2).

23. Taylor RM, Franck LS, Gibson F, Donaldson N, Dhawan A. Study of factors affecting health-related quality of life in adolescents after liver transplantation. American Journal Transplantation. 2009;9:1179-88.

24. Matos MMCSP. Impacto da doença e do transplante hepático na qualidade de vida de doentes com cirrose: estudo exploratório. Lisboa, Portugal: Universidade Aberta; 2007.

25. Corruble E, Barry C, Varescon I, Falissard B, Castaing D, Samuel D. Depressive symptoms predict long-term mortality after liver transplantation. Journal of psychosomatic research. 2011;71(1):32-7.

26. Ferreira CHT, Vieira SMG, Silveira TRd. Transplante hepático. Jornal de pediatria Vol 76, supl 2 (2000), p S198-S208. 2000.

27. Felício HCCD. Manual de orientações para pacientes e familiares: transplante de fígado. São José do Rio Preto: Faculdade de Medicina da Universidade de São José do Rio Preto; 2007.

28. Dobbels F, Hames A, Aujoulat I, Heaton N, Samyn M. Should we retransplant a patient who is non-adherent? A literature review and critical reflection. Pediatric transplantation. 2012;16(1):4-11.
29. O'Carroll RE, Couston M, Cossar J, Masterton G, Hayes PC. Psychological outcome and quality of life following liver transplantation: A prospective, national, single-center study. Liver transplantation. 2003;9(7):712-20.

30. Sahin Y, Virit O, Demir B. Depression and anxiety in parents of children who are candidates for liver transplantation. Arquivos de Gastroenterologia. 2016;53(1):25-30.

31. Anton MC, Piccinini CA. O impacto do transplante hepático infantil na dinâmica familiar. Psicologia: Reflexão e Crítica. 2010;23(2):187-97.

32. Suris J-C, Michaud P-A, Viner R. The adolescent with a chronic condition. Part I: developmental issues. Archives of disease in childhood. 2004;89(10):938-42.

33. Sherlock S, Silva JD. Doenças do fígado e do sistema biliar: Guanabara Koogan; 1991.

34. Alonso EM. Quality of life for pediatric liver recipients. Liver Transplantation. 2009;15(S2).

35. Bernheimer LP, Weisner TS. "Let me just tell you what I do all day...": The family story at the center of intervention research and practice. Infants \& Young Children. 2007;20(3):192-201.

36. Denny B, Beyerle K, Kienhuis M, Cora A, Gavidia-Payne S, Hardikar W. New insights into family functioning and quality of life after pediatric liver transplantation. Pediatric transplantation. 2012;16(7):711-5.

37. Calinescu AM, McLin VA, Belli D, Wildhaber BE. Psycho-social outcome in liver transplanted children: beware of emotional selfassessment! Italian journal of pediatrics. 2012;38(1):1.

38. Moretto MLT. O psicanalista num programa de transplante de fígado: a experiência do "outro em si". São Paulo.: Universidade de São Paulo; 2006.

39. Sá E, editor Um estranho coração2012: Comunicação oral apresentada no XI Congresso Luso-Brasileiro de Transplantação, Hotel Vila Galé.

40. Tavares EMAMV. A vida depois da vida: reabilitação psicológica e social na transplantação de órgãos. Análise Psicológica. 2004:765-77.

41. Abrunheiro LMM, Perdigoto R, Sendas S. Avaliação e acompanhamento psicológico pré e pós-transplante hepático. Psicologia, Saúde \& Doenças. 2005;6(2):139-43.

42. Gruttadauria S. Psychological evaluation and follow-up in liver transplantation. World J Gastrol. 2009;15(6):694-6.

\section{AGRADECIMENTOS}

\section{Ao Programa de Pós-Graduação da Faculdade de Ciências Médicas através do departamento de Ciências Aplicadas à Cirurgia.}

Ao Serviço de Transplante Hepático da Universidade Estadual de Campinas.

À Fundação CAPES (Comissão de Aperfeiçoamento de Pessoal do Nível Superior) que concedeu Bolsa de Complementação Financeira para nível Doutorado. 